\title{
What Is the Best for Colon Preparation: Single-Dose, Split-Dose or Add-ons to Polyethylene Glycol?
}

\author{
UjJal Poddar, * SRinivas SRinidhi Vadlapudi \\ Department of Pediatric Gastroenterology, Sanjay Gandhi Post Graduate Institute of Medical Sciences, \\ Raebareli Road, Lucknow, Uttar Pradesh. \\ *ujjalpoddar@hotmail.com
}

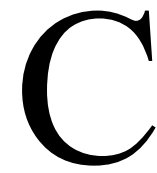

olonoscopy is an important diagnostic and therapeutic modality used in pediatric practice. Chronic bloody diarrhea, bleeding per rectum, surveillance for polyposis syndromes, obtaining tissue biopsies for pathologies such as intestinal tuberculosis and Crohn's disease, and documenting endoscopic mucosal healing in the above conditions are common indications for colonoscopy. Adequate bowel preparation is the essential step in colonoscopy for proper visualization of the mucosa to identify these lesions. It has been estimated that one-third of the colonoscopies are done in suboptimal preparation [1]. In adults, cleanliness of bowel preparation is assessed by Ottawa Bowel Preparation Scale (OBPS). Other commonly used scales in pediatric practice include Aronchik Scale and Boston Bowel Preparation Scale (BBPS) [2]. While Aronchik scale is a global assessment scale, OBPS and BBPS rate the bowel preparation per each colonic segment (such as right colon, transverse colon and left colon). With technological advancement, use of artificial intelligence systems has begun. A software "ENDOANGEL" assesses bowel preparation using BBPS while withdrawing the colonoscope; accuracy was found to be greater than experienced endoscopists [3]. However, it needs further validation studies. In the absence of a universal protocol of bowel preparation, North American Society of Pediatric Gastroenterology, Hepatology and Nutrition (NASPGHAN) endoscopy and procedures committee provided a clinical report in 2014 to guide pediatric bowel preparations for colonoscopy [1].

Polyethylene glycol (PEG), an osmotic laxative, is one of the most commonly used agents for bowel preparation. Various centers use PEG alone or in combination with other agents. The drawback of using PEG is that larger volumes are required. To overcome the issue of using larger volumes in a single go, split-dose regimen has been preferred in adults. NASPGHAN endoscopy and procedures committee had advocated the use of PEG plus electrolytes alone, or in combination with bisacodyl, as a one- or two-day regimen. PEG plus electrolytes when used alone, can be administered as a single or split-dose regimen. Tripathi, et al. [4] have shown better efficacy and tolerability (lesser side effects and improved sleep pattern) of using PEG in a split-dose regimen even in children. The study had a good inter-observer agreement of the findings. Longer regimens of up to four days have also been used [5]. Many practitioners add other osmotic laxatives like magnesium oxide, glycerol or stimulant laxatives such as sodium picosulphate or bisacodyl to enhance bowel preparation [1]. European Society of Gastrointestinal Endoscopy (ESGE) 2019 Guidelines advise the use of low residue diet prior to colonoscopy [6]. Federal Drug Administration (FDA) has recently approved the use of Pure-Vu system, which enhances the bowel preparation quality [7]. It uses a mixture of water and air to break up the fecal matter similar to that of a flush pump. HyGleaCare is another novel system which helps in bowel preparation pre-procedure. It contains a nozzle which infuses a steady stream of warm water that breaks the stool [2]. It is unlikely that small children will cooperate with the use of this device.

In this issue of Indian Pediatrics, a randomized trial by Hein, et al. [8] evaluated 129 children assigned to one of the two bowel preparation strategies by block randomization technique. One group received PEG plus bisacodyl and the other group received PEG plus glycerol enema. Researchers and endoscopists were blinded to the regimens followed. Primary outcome measure was the efficacy of bowel preparation that was assessed by BBPS and the secondary outcome measures were tolerability, acceptability and compliance to the regimens used. No difference was found in the rates of successful bowel preparation using the two regimens. However, side effects such as nausea and bloating were found to be significantly higher in patients receiving PEG plus glycerol. Compliance to therapy was found to be better in patients receiving PEG plus bisacodyl. There was better tolerance to PEG plus bisacodyl in the 
form of willingness to repeat the process. Overall, the use of PEG plus bisacodyl was found to be more acceptable. Strengths of the study include the fact that it was a randomized trial, though open label. The main drawback of the study is that the volume of PEG used in the two regimens was different; $50 \mathrm{~mL} / \mathrm{kg}$ in the $\mathrm{PEG}$ plus bisacodyl group and $70 \mathrm{~mL} / \mathrm{kg}$ in PEG plus glycerol enema group. As has been discussed above, the side effects such as nausea, bloating and poor compliance are related to the use of higher volume of PEG in PEG plus glycerol enema group. The second issue was use of glycerol enema twice in the latter group. Obviously, acceptability of a regimen will be poor if an invasive method (such as rectal enema) is used as compared to oral tablet. Whether all the colonoscopies were performed by a single pediatric endoscopist or multiple persons was not highlighted, and if there was any inter-observer bias was not clearly mentioned. Despite these drawbacks, the article has shown that the dose of PEG can be lowered by adding a stimulant laxative to achieve a good bowel preparation with fewer side effects. Ideally, comparisons should have been made between low dose PEG alone and low dose PEG plus bisacodyl to obviate the above-mentioned drawbacks.

To conclude, in children, appropriate bowel preparation is especially needed due to issues arising from sedation and technical difficulties, which makes repeating the procedure a difficult experience for the patients and attendants. There are various regimens of bowel preparation. PEG alone used as a split-dose regimen is a safe and efficacious regimen validated by a randomized controlled trial in children [4]. There is an unmet need to have a larger randomized controlled trial to compare low dose PEG with low dose PEG plus and stimulant laxative.

Funding: None. Competing interests: None.

\section{REFERENCES}

1. Pall H, Zacur GM, Kramer RE, et al. Bowel preparation for pediatric colonoscopy: report of the NASPGHAN endoscopy and procedures committee. J Pediatr Gastroenterol Nutr. 2014;59:409-16.

2. Mamula $P$, Nema N. Bowel preparation for pediatric colonoscopy. Front Pediatr. 2021;9:705624.

3. Zhou J, Wu L, Wan X, et al. Anovel artificial intelligence system for the assessment of bowel preparation (with video). Gastrointest Endosc. 2020;91:428-435.e2.

4. Tripathi PR, Poddar U, Yachha SK, Sarma MS, Srivastava A. Efficacy of single- versus split-dose polyethylene glycol for colonic preparation in children: a randomized control study. $\mathrm{J}$ Pediatr Gastroenterol Nutr. 2020;70:e1-6.

5. Lightdale JR, Acosta R, Shergill AK, et al. Modifications in endoscopic practice for pediatric patients. Gastrointest Endosc. 2014;79:699-710.

6. Hassan C, East J, Radaelli F, et al. Bowel Preparation for Colonoscopy: European Society of Gastrointestinal Endoscopy (ESGE) Guideline - Update 2019. Endoscopy. 2019;51:775-94.

7. van Keulen K, Neumann H, Schattenberg J, et al. Anovel device for intracolonoscopy cleansing of inadequately prepared colonoscopy patients: a feasibility study. Endoscopy. 2019;51:85-92.

8. Hein PT, Thoi VH, Ha NT, Kalach N. Effectiveness of two regimens for colon cleansing using polyethylene glycol 4000: a randomized open label trial. Indian Pediatr. 2021;58:1119-23. 